\title{
Un orchestre allostérique
}

\section{Jean-Pierre Changeux}

J.P. Changeux : Cnrs URA 2182, Institut Pasteur, 25, rue du Docteur-Roux, 75724 Paris Cedex 15, France.

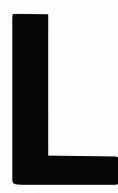

e second millénaire débute par une avancée spectaculaire dans notre connaissance des êtres vivants: la séquence complète du génome de plusieurs bactéries, du ver Caenorhabditis elegans, de la drosophile, de l'homme enfin. Les commentaires vont bon train. Pour les uns, tout est dit: on connaît désormais l'intégralité des protéines et des acides nucléiques présents dans le corps humain; pour les autres, tout reste à faire, puisque nous ne connaissons que les mots du dictionnaire et que le poème reste à écrire.

Le biologiste moléculaire, et plus encore le neurobiologiste, souligneront avec sagesse que les centres d'intérêt, c'est-à-dire les thématiques dans lesquelles surgiront de nouvelles connaissances - se déplacent brutalement de l'unidimensionnel au tridimensionnel. Cela ne signifie en aucune manière que la linéarité du génome et la co-linéarité de ses déterminants soient mises en cause. La règle, "un gène-une enzyme " $n^{\prime} a$ rien perdu de sa véracité. Mais d'un gène, on passe à 70000 . Et cela pose, avec urgence, la signification fonctionnelle de tous ces gènes.
Premier voyage obligé donc dans le tridimensionnel: comment ces séquences linéaires se replient-elles en une molécule définie possédant une fonction définie, par exemple enzymatique ou réceptrice. La biophysique des protéines et les acides nucléiques, des lipides et des polysacharides, les études cristallographiques (gros équipements à l'appui) progressent, pas à pas. Dans un univers de structures moléculaires d'une telle diversité, des airs de famille simplifieront l'analyse morphologique. Mais seul l'examen dans le détail, à l'atome près, conduira à la fonction précise. Et encore, il ne s'agit que d'un premier pas.

Le monde des interactions entre molécules est encore plus complexe. Ce numéro de médecine/sciences est une première fenêtre sur une gigantesque combinatoire qu'il va falloir déchiffrer. Dans les années 1960, une première étape a porté sur la signification des interactions que certaines protéines forment avec elles-mêmes. Une même sous-unité peut $\mathrm{s}^{\prime}$ assembler en un édifice clos et fini que l'on qualifie d'oligomère. Exemple: I'hémoglobine est un tétramère; le récepteur de l'acétylcholine un pentamère; beaucoup de facteurs de transcription des dimères. A quoi ces interactions moléculaires élémentaires peuventelles servir ? A consolider l'édifice ou, au contraire, à le rendre plus flexible? L'étude de plusieurs enzymes régulateurs bactériens et de l'hémoglobine montra que l'organisation en structure oligomérique rend possible la mise en place $d^{\prime}$ interactions fonctionnelles entre sites distincts désormais qualifiées d'allostériques. Le mécanisme s'applique aux récepteurs de neurotransmetteurs liés à des canaux ioniques. Il s'applique également, semble-t-il - découverte récente inattendue - aux récepteurs couplés aux protéines $G$ (voir l'article de M. Bouvier et al., p. 644 de ce numéro). L'oligomère - microcristal moléculaire - se prête aux effets coopératifs. On les note lors de la fixation de plusieurs molécules d'oxygène sur une même molécule d'hémoglobine ou, d'une manière générale, lorsqu'un signal régulateur déclenche une réaction physiologique dès qu'il franchit un seuil de concentration critique.

Le propos d'aujourd'hui est plus ambitieux encore. Il se situe à un niveau d'organisation plus élevé, ouvrant la voie aux multiples combinaisons créées par les interactions entre molé- 
cules protéiques différentes, oligomériques ou non. Des échafaudages protéiques de nature insoupçonnée peuvent se construire avec des protéines chaperones (voir l'article de M.Morange, p. 630 de ce numéro), des protéines $G$ petites ou grosses, des canaux ioniques (sans parler des protéines du cytosquelette, et des protéines contractiles). Des adapteurs moléculaires aux domaines d'interactions moléculaires spécialisés désormais bien connus (voir l'article de I. Broutin et A. Ducruix, p. 611 de ce numéro) créent de multiples liens structuraux pouvant servir à $d^{\prime}$ innombrables voies de signalisation intracellulaire. De la membrane au noyau, des réseaux $d$ 'interaction fonctionnelle se mettent en place. La cellule n'est plus un "sac d'enzyme " comme cela se disait dans les années 1950. Bien au contraire. Des foyers d'interactions moléculaires s'organisent en réseaux fonctionnels, tant au niveau de la formation du complexe d'initiation de la transcription (voir l'article de F. Coin et J.M. Egly, p. 593 de ce numéro), de réparation de I'ADN, de la traduction (voirl'article de F. Poulin et S. Pyronnet, p. 617 de ce numéro), de la formation des nucléosomes (voir l'article de A. Taddei et al., p. 603 de ce numéro), que de la dégradation des protéines (voir l'article de $\mathrm{O}$. Coux et M. Piechaczyk, p. 623 de ce numéro). Des architectures dynamiques supramacromoléculaires, canalisent, coordonnent la biosynthèse, l'assemblage, le transport et le triage (voir l'article de P. Cosson et F. Letourneur, p. 635 de ce numéro) des principaux composants de la vie de la cellule, y compris la réplication de son ADN et sa division. Le cas de la cellule nerveuse avec ses multiples prolongements axonique et dendritique, son cytosquelette et son "manteau d'arlequin" de récepteurs l'illustre de manière plus saisissante encore.

Une image pour conclure. Les chaînes d'interactions moléculaires en cascade ne forment pas une réticulation rigide qui emprisonnerait la cellule de l'intérieur. D'abord, la flexibilité structurale conférée en particulier par des interactions allostériques à des nœuds critiques de ce réseau confère à la cellule une capacité réactionnelle dont l'éventail des possibilités sera, peut-être, un jour calculable. Plus important encore, ce réseau régulateur interne à la cellule s'intègre par le truchement de ses récepteurs membranaires de surface à un réseau $d$ 'interactions régulatrices entre cellules. Un "orchestre" se forme spontanément entre les divers composants de la cellule. Il se mêle à celui qui se développe entre cellules. La connexion entre réseaux intra- et extracellulaire devient un centre d'intérêt essentiel dans la compréhension de la morphogénèse embryonnaire $[1,2]$. Cette interaction est plus critique encore dans les fonctions d'apprentissage et de mémoire du système nerveux central. Le postgénome à trois dimensions a un brillant avenir

\section{RÉFÉRENCES}

1. Kerszberg $M$, Changeux JP. A model for reading morphogenetic gradients: autocatalysis and competition at the gene level. Proc Natl Acad Sci USA 1994; 91 : 5823-7.

2. Kerszberg $M$, Changeux JP. A simple molecular model of neurulation. BioEssays $1998 ; 20$ : 758-70.

\section{TIRÉS À PART}

J.P. Changeux. 


\section{-ms 2000 \\ Summary}

Transport and protein sorting in eukaryotic cells

This review describes fundamental cellular mechanisms involved in intracellular transport. The ubiquitous role of transport vesicles allows similar mechanisms to participate in many different steps of intracellular transport. In each case, a different cytoplasmic coat polymerizes on the membrane of the budding vesicle, and interacts with specific signals located in the cytoplasmic domains of membrane proteins which must be concentrated in the vesicle. The number of characterized cytoplasmic sorting signals is increasing rapidly, as well as the number of identified coats. Intracellular sorting can be perturbed by inherited mutations and can result in pathological situations such as cystic fibrosis or familial hypercholesterolemia. Viruses can also use or divert sorting mechanisms of eucaryotic cells, which are essential for their efficient propagation. Therefore, deciphering the cellular machinery responsible for intracellular transport and sorting of proteins is essential for a better understanding of many pathological situations. 